\title{
Charge Transport and Magnetism in $\mathrm{Tm}_{0.03} \mathrm{Yb}_{0.97} \mathrm{~B}_{12}$
}

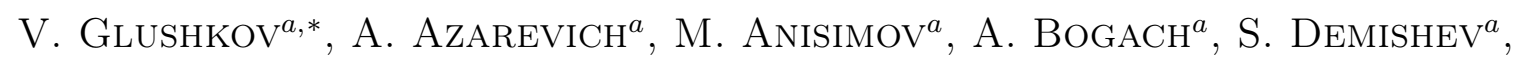 \\ A. Dukhnenko ${ }^{b}$, V. Filipov ${ }^{b}$, K. Flachbart ${ }^{c}$, S. GabÁni $^{c}$, S. Gavrilkin $^{d}$, M. Kondrin $^{e}$, \\ N. Shitsevalova ${ }^{b}$ AND N. SluChankO ${ }^{a}$ \\ ${ }^{a}$ Prokhorov General Physics Institute of RAS, Vavilov Str. 38, 119991 Moscow, Russia \\ ${ }^{b}$ Frantsevich Institute for Problems of Materials Science, NAS, Krzhyzhanovsky Str. 3, 03680 Kiev, Ukraine \\ ${ }^{c}$ Institute of Experimental Physics, SAS, Watsonova 47, 04001 Košice, Slovakia \\ ${ }^{d}$ Lebedev Physical Institute of RAS, Leninskii pr. 53, 119991 Moscow, Russia \\ ${ }^{e}$ Vereshchagin Institute of High Pressure Physics of RAS, Troitsk, 142190 Moscow, Russia
}

\begin{abstract}
Transport and magnetic properties of polycrystalline $\mathrm{Tm}_{0.03} \mathrm{Yb}_{0.97} \mathrm{~B}_{12}$ samples were investigated at temperatures $1.8-300 \mathrm{~K}$ in magnetic fields up to $9 \mathrm{~T}$. The activated behavior of resistivity, the Hall coefficient and thermopower is described in terms of a narrow gap $\varepsilon_{g} \approx 16.6 \mathrm{meV}$, which controls the charge transport in $\mathrm{Tm}_{0.03} \mathrm{Yb}_{0.97} \mathrm{~B}_{12}$ at $T>40 \mathrm{~K}$. The maximum of magnetic susceptibility found at $50 \mathrm{~K}$ is shown to be induced by a spin gap $\Delta \approx 4.7 \mathrm{meV}$ being close to the half of the spin fluctuation energy in $\mathrm{YbB}_{12}$. Large diffusive thermopower $S=A T, A=-29.1 \mu \mathrm{V} / \mathrm{K}^{2}$ and the Pauli susceptibility $\chi_{0} \approx 7.2 \times 10^{-3} \mathrm{emu} / \mathrm{mol}$ found below $20 \mathrm{~K}$ seem to be associated with the many-body resonance, which corresponds to states with an enhanced effective mass $m^{*} \approx 250 m_{0}$ $\left(m_{0}\right.$ - free electron mass). The effective parameters of magnetic centers and the analysis of anomalies favor the nonequivalent states of substitute Tm ions.
\end{abstract}

DOI: 10.12693/APhysPolA.131.985

PACS/topics: 72.15.Gd, 72.15.Jf, 71.27.+a

\section{Introduction}

The nature of the narrow gap $\left(\varepsilon_{g} \approx 17.8 \mathrm{meV}[1,2]\right)$ in $\mathrm{YbB}_{12}$, which shares the place between antiferromagnetic metal $\mathrm{TmB}_{12}[3]$ and superconducting $\mathrm{LuB}_{12}[4]$ in the set of rare-earth dodecaborides $\mathrm{RB}_{12}$, stays a subject of discussions $[1-3,5-11]$. The ground state of $\mathrm{YbB}_{12}$ identified usually as the Kondo insulator [1] seems to have a non-trivial topology of the band structure resulting in surface conductivity [5]. However, studies of Ludoped and $\mathrm{Zr}$-doped $\mathrm{YbB}_{12}$ show that the gap in the $\mathrm{YbB}_{12}$ band spectrum is local and is not influenced by the onset of long-range coherence [6,7]. Recent studies of $\mathrm{Tm}_{x} \mathrm{Yb}_{1-x} \mathrm{~B}_{12}$ single crystals [8-10] pointed out that the rise of $\mathrm{Yb}$ content results in a metal-insulator transition, a bulk narrow many-body resonance $(\Delta \approx 6 \mathrm{meV})$ appears at the Fermi level. The band spectrum renormalization seen from the thermopower enhancement (from $S=-2 \mu \mathrm{V} / \mathrm{K}$ for $\mathrm{TmB}_{12}$ up to $S=-230 \mu \mathrm{V} / \mathrm{K}$ for $\mathrm{Yb}_{0.81} \mathrm{Tm}_{0.19} \mathrm{~B}_{12}$ [8]) is suggested to be induced by the $\mathrm{Yb}^{3+}-\mathrm{Yb}^{3+}$ dimer formation. This assumption may be proved by a study of Yb-rich samples $(x<0.19)$, which were not available for the transport studies up to now $[9-11]$.

\section{Experimental methods}

To shed more light on the nature of the ground state of such a system, transport and magnetic properties of the

\footnotetext{
*corresponding author; e-mail: glushkov@lt.gpi.ru
}

$\mathrm{Tm}_{0.03} \mathrm{Yb}_{0.97} \mathrm{~B}_{12}$ substitutional solid solution were studied. High purity polycrystalline $\mathrm{Tm}_{0.03} \mathrm{Yb}_{0.97} \mathrm{~B}_{12}$ samples were grown by crucibleless inductive zone melting in argon atmosphere. Any secondary phases were excluded by X-ray diffraction analysis. The real thulium content in the solid solution estimated from EPMA study $(x \approx 0.04)$ was found to exceed slightly the nominal one. A five probe method was used to measure resistivity and Hall effect at temperatures 2-300 K in magnetic fields up to $8.2 \mathrm{~T}$. The Seebeck coefficient was studied at temperatures 3-300 K by the original 4-probe technique with a step-by-step temperature gradient sweeping at fixed temperature [8]. The temperature and field dependences of magnetization were measured with the help of Quantum Design PPMS-9 setup.

\section{Results and discussion}

Transport properties of $\mathrm{Tm}_{0.03} \mathrm{Yb}_{0.97} \mathrm{~B}_{12}$ are summarized in Fig. 1. Lowering of temperature results in a monotonous increase of resistivity (Fig. 1a), which changes from $\rho(300 \mathrm{~K}) \approx 440 \mu \Omega \mathrm{cm}$ to $\rho(2 \mathrm{~K}) \approx$ $9.6 \mathrm{~m} \Omega \mathrm{cm}$. The large inverse resistivity ratio IRR $=$ $\rho(2 \mathrm{~K}) / \rho(300 \mathrm{~K}) \approx 22$ as compared to $\mathrm{IRR} \approx 9.3$ for $\mathrm{Tm}_{0.19} \mathrm{Yb}_{0.81} \mathrm{~B}_{12}$ single crystal [9] proves the high quality of samples under investigation. At $T>100 \mathrm{~K}$ the resistivity is well described by the thermal activation law $\rho \sim$ $\exp \left(-E_{R} / T\right)$ with a characteristic energy $E_{R} \approx 75.3 \mathrm{~K}$. The $E_{R}$ value is considerably lower than those ones estimated for the Hall constant $\left(E_{\mathrm{H}} \approx 96.7 \mathrm{~K}\right)$ and the Seebeck coefficient $\left(E_{\mathrm{S}} \approx 173 \mathrm{~K}\right)$. The $E_{\mathrm{H}}$ value gives a correct estimation of the gap size in $\operatorname{Tm}_{0.03} \mathrm{Yb}_{0.97} \mathrm{~B}_{12}$ $\left(\varepsilon_{g}=2 E_{\mathrm{H}} \approx 16.6 \mathrm{meV}\right)$. The same signs of the Hall and 
Seebeck effects (Fig. 1c,d) prove the major electron contribution to charge transport while the negative magnetoresistance $\Delta \rho / \rho=(\rho(H)-\rho(0)) / \rho(0)$ points to a dominant magnetic scattering of charge carriers. However, the discrepancy between the $E_{\mathrm{H}}$ and $E_{\mathrm{S}}$ values $\left(E_{\mathrm{H}}<E_{\mathrm{S}}\right)$ cannot be explained by the difference between the mobilities of holes and electrons in the intrinsic semiconductor model.

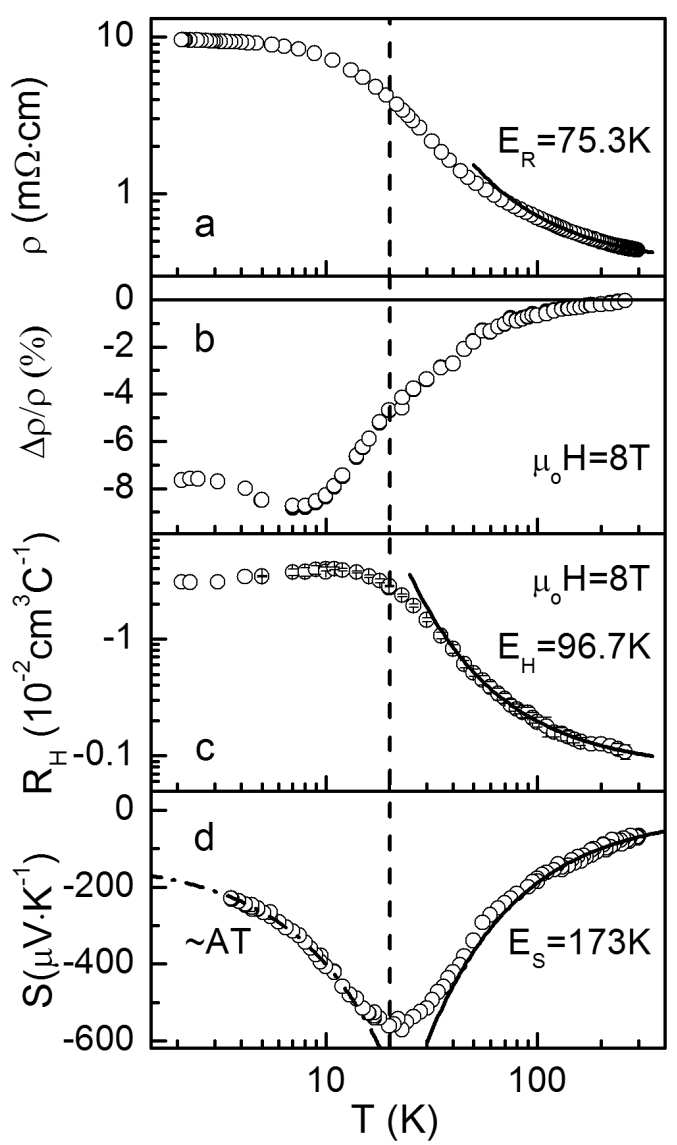

Fig. 1. Resistivity $\rho$ (a), magnetoresistance $\Delta \rho / \rho$ (b), the Hall constant $R_{\mathrm{H}}$ (c) and thermopower $S$ (d) of $\mathrm{Tm}_{0.03} \mathrm{Yb}_{0.97} \mathrm{~B}_{12}$. Solid lines correspond to the activation asymptotics (see text). The dash-dotted line in part (d) shows the linear $S(T)$ fit with $A=-29.1 \mu \mathrm{V} / \mathrm{K}^{2}$. The vertical dashed line marks the position of $S(T)$ minimum (see also Fig. 3).

The saturation of resistivity and Hall constant below $10 \mathrm{~K}$ (Fig. 1a,c) is followed by an emergent feature of thermopower, which passes through a minimum $S \approx-560 \mu \mathrm{V} / \mathrm{K}$ at $T=20 \mathrm{~K}$ and rises as $S \sim A T$ with $A=-29.1 \mu \mathrm{V} / \mathrm{K}^{2}$ when temperature decreases (Fig. 1d). The extreme value of the Hall constant at $T \approx 10 \mathrm{~K}$ (Fig. 2c) appears due to contribution from the anomalous Hall effect identified clearly from the field dependences of the Hall resistivity. This contribution does not exceed $5.6 \mu \Omega \mathrm{cm}$ and will be discussed elsewhere.

The temperature dependence of the magnetic susceptibility calculated from $M(T, 0.1 T)$ magnetization data as $\chi=M / H$ shows a non-monotonous behavior with low- temperature upturn (Fig. 2a). At $T>40 \mathrm{~K}$ the $\chi(T)$ data can be well fitted by the spin gap model $\chi_{\mathrm{S}}(T)=$ $\chi_{S 0}+C_{\mathrm{S}} / T \exp (-\Delta / T)$ applied earlier for the relative compound $\mathrm{SmB}_{6}[12]$. The spin gap size $\Delta \approx 54.7 \mathrm{~K}$ is approximately equal to the half of the spin fluctuations temperature in $\mathrm{YbB}_{12}\left(T_{s f} \approx 100 \mathrm{~K}\right)$ [11] and is comparable with the binding energies of in-gap manybody states $\left(E_{a}=65 \pm 10 \mathrm{~K}\right)$ detected in $\mathrm{Tm}_{1-x} \mathrm{Yb}_{x} \mathrm{~B}_{12}$ $(x<0.19)$ [9]. The Curie constant $C_{s} \approx 1.77 \mathrm{emu} \mathrm{K} / \mathrm{mol}$ corresponds to the effective moment $\mu_{\text {eff }} \approx 3.8 \mu_{\mathrm{B}}\left(\mu_{\mathrm{B}}\right.$ - Bohr magneton), which is considerably lower than the respective free $\mathrm{Yb}^{3+}$ ion value $\mu_{\mathrm{eff}} \approx 4.5 \mu_{\mathrm{B}}$.

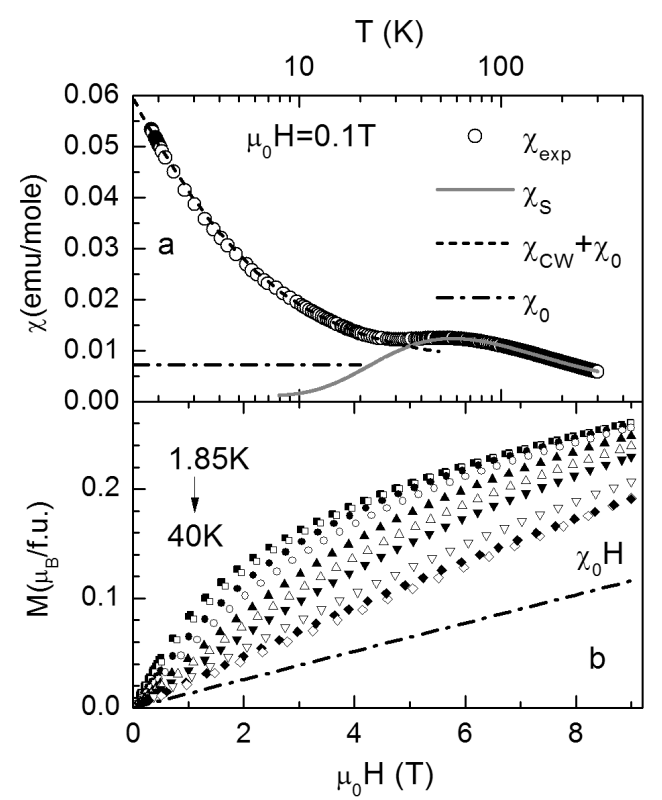

Fig. 2. (a) Molar susceptibility $\chi(T)$ of $\mathrm{Tm}_{0.03} \mathrm{Yb}_{0.97} \mathrm{~B}_{12}$ calculated from the $M(T, 0.1 T)$ magnetization data. Solid and dashed lines represent the fits within spin gap and the Curie-Weiss models (see text). (b) Isothermal magnetization $M\left(H, T_{0}\right)$ of $\mathrm{Tm}_{0.03} \mathrm{Yb}_{0.97} \mathrm{~B}_{12}$ measured at $T_{0}=1.85,2.3,3.1,4.2$, $6,8,10,15,20$, and $40 \mathrm{~K}$. Dash-dotted lines in parts (a) and (b) show the contributions from low-temperature Pauli susceptibility $\chi_{0} \approx 7.2 \times 10^{-3} \mathrm{emu} / \mathrm{mol}$.

Below $20 \mathrm{~K}$ magnetic susceptibility follows the CurieWeiss law $\chi_{\mathrm{CW}}=C_{0} /(T-\Theta)$ with $C_{0} \approx 0.13 \mathrm{emu} \mathrm{K} / \mathrm{mol}$ and $\Theta \approx-1.0 \mathrm{~K}$, which is biased by the temperature independent contribution $\chi_{0} \approx 7.2 \times 10^{-3} \mathrm{emu} / \mathrm{mol}$ (Fig. 2a). The $\chi_{0} H$ term agrees well with the high field trend of the $M(H, T<4 \mathrm{~K})$ data (Fig. 2b). The correct estimation of $\chi_{\mathrm{CW}}$ and $\chi_{0}$ is also proved by the scaling of saturated magnetization $M-\chi_{0} H$ as a function of $H /(T-\Theta)$ being valid for $T<20 \mathrm{~K}$ (not shown here). Note that a similar procedure has been successfully applied to separate different contributions to the magnetization of the $\mathrm{Tm}_{1-x} \mathrm{Yb}_{x} \mathrm{~B}_{12}$ solid solutions for $x<0.19[10]$. The straightforward calculation for $\mathrm{Tm}_{0.03} \mathrm{Yb}_{0.97} \mathrm{~B}_{12}$ results in the saturated moment $\mu_{\mathrm{S}} \approx 7.3 \mu_{\mathrm{B}}$ and the effective concentration of centers $N_{0} \approx 0.02$ (per formulae unit). 
The correlated behavior of transport and magnetic properties for Yb-rich dodecaborides can be clearly established from the temperature behavior of the difference $\chi(T)-\chi_{\mathrm{CW}}(T)$ (Fig. 3a) and from effective parameters of charge carriers (Fig. 3b,c). Indeed, the electron concentration estimated from the Hall constant as $n=\left(R_{\mathrm{H}} e\right)^{-1}$ can be well fitted by the combination of a thermally activated contribution $n(T) / n_{R E}=n_{1} \exp \left(-E_{\mathrm{H}} / T\right)$ with $n_{R E}=9.6 \times 10^{21} \mathrm{~cm}^{-3}$ and $n_{1}=0.92$ and a temperature independent addition $n_{0}=0.016$ per rare-earth ion (Fig. 3c). The crossover temperature $T_{0} \approx 20 \mathrm{~K}$ matches perfectly the positions of the electron mobility maximum (Fig. 3b) and the maximal amplitude of the Seebeck effect (Fig. 1d). Besides, the $\Delta \chi=\chi(T)-\chi_{\mathrm{CW}}(T)$ contribution increases rapidly below $T_{0}$ approaching the estimated $\chi_{0}$ value (Fig. 3a).

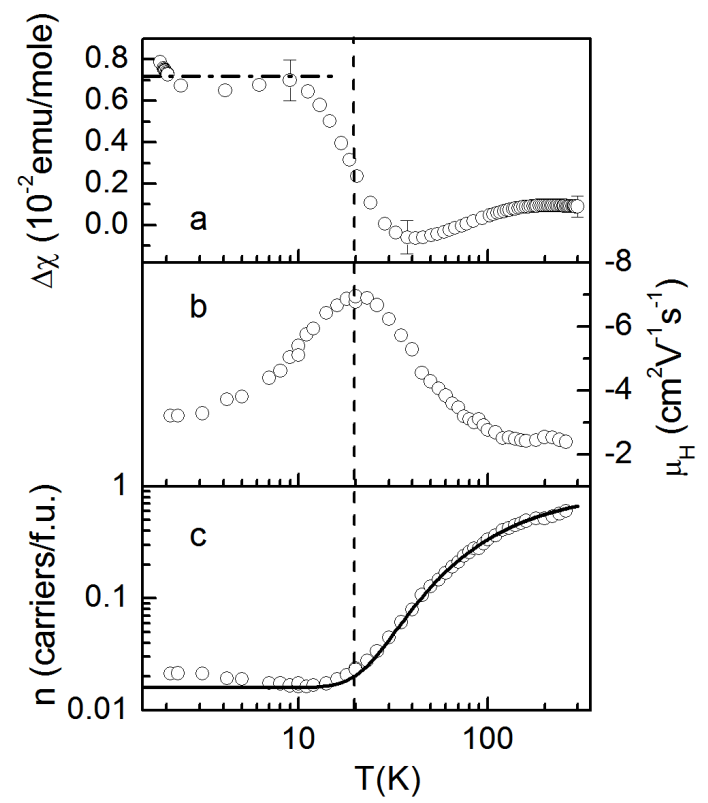

Fig. 3. The difference $\Delta \chi=\chi(T)-\chi_{\mathrm{CW}}(T)$ (a), the Hall mobility $\mu_{\mathrm{H}}(\mathrm{b})$, and concentration of charge carriers per formula unit $n$ (c) in $\mathrm{Tm}_{0.03} \mathrm{Yb}_{0.97} \mathrm{~B}_{12}$. The dash-dotted line in part (a) shows the value of $\chi_{0}$ (see caption to Fig. 2). The solid line in part (c) corresponds to the $n(T) / n_{R E}=n_{0}+n_{1} \exp \left(-E_{\mathrm{H}} / T\right)$ fit with $n_{0}=0.016$ and $n_{1}=0.92$. The vertical dashed line marks the position of $\left|\mu_{\mathrm{H}}(T)\right|$ maximum (see also Fig. 1)

In our opinion, the low temperature anomalies of charge transport and magnetic properties of $\mathrm{Tm}_{0.03} \mathrm{Yb}_{0.97} \mathrm{~B}_{12}$ can be well understood in terms of a temperature induced transformation of the band spectrum discussed earlier in [8-10]. Indeed, a straightforward calculation of the density of states at the Fermi level using standard expressions for diffusive thermopower $A T$ and the Pauli susceptibility $\chi_{0}$ results in $N\left(\varepsilon_{F}\right) \approx 4.9 \times$ $10^{35} \mathrm{erg}^{-1} \mathrm{~cm}^{-3}$ and $N\left(\varepsilon_{F}\right) \approx 5.7 \times 10^{35} \mathrm{erg}^{-1} \mathrm{~cm}^{-3}$, respectively. Within the single electron model these values correspond to an extremely large effective mass of charge carriers $m^{*} \approx 250 m_{0}$. The estimated relaxation time $\tau=m^{*} \mu_{\mathrm{H}} / e \approx 0.6 \mathrm{ps}$ agrees well with inverse valence fluctuation rate $\tau \approx 0.4$ ps estimated from optical and neutron studies [2, 11]. Finally, the very good correlation between the sum $N_{0}+n_{0} \approx 0.036$ and the Tm concentration $(\approx 0.04)$ favors the nonequivalent states of substitute ions, which may appear due to their various positions in respect of the ytterbium dimers $[9,10]$. However, an extended study of the $4 f-5 d$ hybridization effects in Yb-rich compounds is required to prove this suggestion.

\section{Acknowledgments}

The support from RFBR project 15-02-03166 and from project VEGA 2/0032/16 are acknowledged.

\section{References}

[1] F. Iga, N. Shimizu, T. Takabatake, J. Magn. Magn. Mater. 177-181, 337 (1998).

[2] B. Gorshunov, P. Haas, O. Ushakov, M. Dressel, F. Iga, Phys. Rev. B 73, 045207 (2006).

[3] N. Sluchanko, L. Bogomolov, V. Glushkov, S. Demishev, M. Ignatov, Eu. Khayrullin, N. Samarin, D. Sluchanko, A. Levchenko, N. Shitsevalova, K. Flachbart, Phys. Status Solidi B 243, R63 (2006).

[4] K. Flachbart, S. Gabáni, K. Gloos, M. Meissner, M. Opel, Y. Paderno, V. Pavlík, P. Samuely, E. Schuberth, N. Shitsevalova, K. Siemensmeyer, P. Szabó, J. Low Temp. Phys. 140, 239 (2005).

[5] H. Weng, J. Zhao, Zh. Wang, Zh. Fang, Xi Dai, Phys. Rev. Lett. 112, 016403 (2014).

[6] F. Iga, S. Hiura, J. Klijn, N. Shimizu, T. Takabatake, M. Ito, Y. Matsumoto, F. Masaki, T. Suzuki, T. Fujita, Physica B 259-261, 312 (1999).

[7] K.S. Nemkovski, P.A. Alekseev, J.-M. Mignot, E.A. Goremychkin, A.A. Nikonov, O.E. Parfenov, V.N. Lazukov, N.Yu. Shitsevalova, A.V. Dukhnenko, Phys. Rev. B 81, 125108 (2010).

[8] N.E. Sluchanko, A.V. Bogach, V.V. Glushkov, S.V. Demishev, K.S. Lyubshov, D.N. Sluchanko, A.V. Levchenko, A.B. Dukhnenko, V.B. Filipov, S. Gabáni, K. Flachbart, JETP Lett. 89, 256 (2009).

[9] N.E. Sluchanko, A.N. Azarevich, A.V. Bogach, V.V. Glushkov, S.V. Demishev, M.A. Anisimov, A.V. Levchenko, V.B. Filipov, N.Yu. Shitsevalova, J. Exp. Theor. Phys. 115, 509 (2012).

[10] A.V. Bogach, N.E. Sluchanko, V.V. Glushkov, S.V. Demishev, A.N. Azarevich, V.B. Filippov, N.Yu. Shitsevalova, A.V. Levchenko, J. Vanacken, V.V. Moshchalkov, S. Gabani, K. Flachbart, J. Exp. Theor. Phys. 116, 838 (2013).

[11] P.A. Alekseev, K.S. Nemkovski, J.-M. Mignot, E.S. Clementyev, A.S. Ivanov, S. Rols, R.I. Bewley, V.B. Filipov, N.Yu. Shitsevalova, Phys. Rev. B 89, 115121 (2014).

[12] V.V. Glushkov, A.V. Kuznetsov, O.A. Churkin, S.V. Demishev, Yu.B. Paderno, N.Yu. Shitsevalova, N.E. Sluchanko, Physica B 378-380, 614 (2006). 